\title{
Construcción de un instrumento para medir el clima organizacional en función del modelo de los valores en competencia
}

\author{
Roberto Hernández \\ Sampieri \\ Escuela Superior de Comercio \\ y Administración, Instituto \\ Politécnico Nacional \\ rhs.rhs@hotmail.com
}

Sergio Méndez Valencia Universidad de Celaya smendez@udec.edu.mx

Ricardo Contreras Soto Universidad de Guanajuato riconsoto@hotmail.com

\section{Resumen}

El presente estudio estableció como objetivo central analizar en el ámbito laboral mexicano un modelo que contextualizara al clima organizacional y lo vinculara con la cultura organizacional. Se eligió al modelo de valores en competencia (MVC) de Kim S. Cameron y Robert E. Quinn, por ser tal vez el marco de referencia con mayor evidencia empírica en lo que respecta a la cultura organizacional. El MVC propone una estructura de cuadrantes de valores para las organizaciones: 1) enfoque interno, flexibilidad (organización familiar o clan); 2) enfoque interno, estabilidad y control (organización jerárquica); 3) enfoque externo, flexibilidad (organización adhocrática); y 4) enfoque externo, estabilidad y control (organización de mercado o metas). Asimismo, este modelo sostiene que las organizaciones presentan una mezcla de valores de todos los cuadrantes. Los resultados, en términos generales, fundamentaron dicho modelo en una muestra heterogénea de doce organizaciones de la región central de México (1 424 casos). Por otra parte, se construyó una primera versión de un instrumento que pretende medir el clima organizacional en función del MVC, el cual demostró capacidad de discriminar entre organizaciones y ser en términos generales válido y confiable.

Palabras clave: cultura organizacional, clima organizacional, modelo de valores en competencia, validación. 


\title{
Building an instrument to measure the organizational climate as a function of the Competing Values Framework
}

\begin{abstract}
The purpose of this study was to explore a model in the Mexican labor field that contextualizes organizational climate and links it with organizational culture. The Competing Values Framework (CVF), by K. S. Cameron \& R. E. Quinn, was chosen due to its importance as the framework with more empirical evidence regarding corporate culture. The CVF proposes a four-quadrant structure for organizations: 1) internal focus, flexibility (clan), 2) internal focus, stability and control (hierarchy), 3) external focus, flexibility (adhocracy), and 4) external focus, stability and control (market). Likewise, it states that organizations present a mix of values from all quadrants. Overall, the findings of the study founded the model on a heterogeneous sample of 12 organizations $(n=1424)$ in the central region of Mexico. In addition to the model, a primer version of an instrument was designed with the intention to measure organizational climate according to the CVF. The instrument proved its capability to discriminate amongst organizations and, in general, its reliability and validity.
\end{abstract}

Keywords: organizational culture, organizational climate, competing values framework, validation.

\section{Introducción}

El presente trabajo constituye un esfuerzo investigativo desde dos perspectivas: la primera conceptual que consiste en relacionar el clima laboral con la cultura organizacional debido a que ambos conceptos han sido ampliamente vinculados en la literatura organizacional, aunque no de manera empírica, ni tampoco utilizando un marco de referencia apropiado; la segunda perspectiva es de naturaleza metodológica, pues busca validar un instrumento que mida las típicas variables del clima laboral encuadradas en un modelo de cultura organizacional, reconociendo las limitaciones de la herramienta (confiabilidad, validez, variables consideradas) y la necesidad de realizar más investigaciones en distintos contextos y muestras. Las dos perspectivas implican los objetivos esenciales del estudio.

Después de revisar varios modelos de cultura organizacional se seleccionó el modelo de valores en competencia (MVC) para enmarcar al clima organizacional y así asociar ambos constructos conceptualmente. Siguiendo el modelo y de acuerdo con la revisión de la literatura, se seleccionaron variables del clima organizacional, las cuales fueron medidas y analizadas dentro del MVC. 
Tal modelo fue elegido por ser un encuadre teórico que aglutina los principales enfoques administrativos y contar con abundante evidencia empírica (Cameron y Quinn, 2011; Maldonado, Martínez y García, 2010; Yu y Wu, 2009; Muro, 2008; Quinn y Rohrbaugh, 1983).

\section{Marco teórico}

\section{El clima organizacional}

El concepto del clima organizacional ha recibido en las últimas décadas una importante atención por parte de psicólogos industriales, administradores del desarrollo empresarial y sociólogos de la organización. Particularmente, a partir del año 2000 recobró un notable interés, lo cual se debe, en gran medida, a que la mayoría de los modelos de comportamiento organizacional se centran en las percepciones del ambiente laboral, referido como el clima organizacional (Patterson et al., 2005). Además de que está correlacionado a una diversidad de resultados empresariales o institucionales (Mamiseishvili, 2011; Kuenzi y Schminke, 2009; Mathieu, Maynard, Rapp y Gilson, 2008).

\section{Definiciones de clima organizacional}

Actualmente, no existe un consenso total sobre una definición única (Parker et al., 2003), aunque el concepto ha inspirado varias descripciones y operacionalizaciones. Desde luego, el clima organizacional es entendido, en términos generales, como una variable interviniente entre el contexto de una organización y la conducta de sus miembros, así como intenta capturar o comprender cómo los empleados experimentan el trabajo en sus empresas o instituciones (King, Hebl, George y Matusik, 2010; Patterson et al., 2005; Brunet, 2002).

La mayoría de las definiciones conceptualiza al clima organizacional o laboral como un conjunto de percepciones de los individuos con respecto a su medio interno de trabajo. Estas percepciones reflejan la interacción entre los elementos individuales y las características y procesos de la organización (Schneider, Ehrhart y Macey, 2011).

McKnight y Webster (2001) consideran al clima organizacional como las percepciones compartidas por los empleados sobre las prácticas y procedimientos de la empresa o institución, incluyendo qué conductas son esperadas y recompensadas. 
Brunet define el clima organizacional como: "las actitudes subyacentes a los valores, a las normas y a los sentimientos que los empleados tienen ante su organización" (2002, p. 55).

De las definiciones se desprende que el clima organizacional está fundamentado en percepciones colectivas del personal con respecto a variables de la organización (como la estructura, las políticas y las prácticas administrativas) y a los procesos humanos que ocurren en la interacción cotidiana dentro de la organización (comunicación, liderazgo, ejercicio de la jerarquía, etcétera).

\section{Análisis del clima organizacional}

La mayoría de los estudios empíricos en este campo han utilizado una unidad de análisis agregada como el grupo de trabajo, el cual se refiere al departamento o la organización (los constructos de clima grupal, departamental u organizacional). Tales climas han sido construidos operacionalmente adicionando puntuaciones de casos al nivel apropiado y utilizando estadísticas de tendencia central para representar al clima en dicho nivel. El argumento racional detrás del análisis agregado de datos individuales en una unidad es la premisa con relación al que las colectividades organizacionales poseen en su propio clima y que éste puede ser identificado mediante la demostración de diferencias significativas entre unidades con respecto al clima y acuerdos reveladores en las percepciones dentro de las unidades (Patterson, Warr y West, 2004).

Para establecer las fronteras de su revisión, los estudios más recientes (por ejemplo, Smith-Crowe, Burke, y Landis, 2003; Parker et al., 2003; Patterson et al., 2005; Ostroff, Kinicki, y Tamkins, 2003) han considerado importante comenzar depurando el concepto y distinguirlo de otros constructos. En su opinión, mucha de la confusión conceptual producida por el uso de múltiples variables complejas puede remediarse clarificando un nivel de teoría, de medida y de análisis (Parker et al., 2003). Para tal clarificación debe partirse de un modelo que encuadre al clima organizacional y en el cual se incluyan y midan variables pertinentes y consistentes con éste (Patterson et al., 2005).

\section{Operacionalización del clima organizacional}

En cuanto a la operacionalización del clima organizacional, podemos decir que existen insuficientes mediciones validadas del constructo (Schneider et al., 2011). 
Esto último es particularmente cierto en el contexto latinoamericano (Hernández Sampieri, 2006; Aralucen, 2003). De acuerdo con Patterson et al. (2005), el clima ha sido más bien descrito consistentemente como las percepciones de los empleados con relación a sus organizaciones, la construcción se ha visto envuelta en definiciones operacionales conflictivas e inconsistencias en su medición.

A través de los años, las dimensiones que han sido identificadas como componentes de esta compleja variable han sido distintas, por lo que diversas visiones emanaron, conduciendo a una confusión y un lento progreso teórico (Brunet, 2002).

Litwin y Stringer (1968) en un estudio pionero y clásico midieron la percepción de los empleados en función de nueve dimensiones: estructura, responsabilidad individual, remuneración, desafío, relaciones sociales, cooperación, estándares, conflictos e identificación con la organización. En sus revisiones sobre la literatura, L. R. James y L. A. James identificaron cinco dominios primarios de las percepciones sobre el ambiente de trabajo: 1) características del trabajo (por ejemplo, autonomía, reto e importancia de la tarea); 2) características del papel o rol laboral (ambigüedad, conflicto y sobrecarga); 3) características del liderazgo (énfasis en las metas, apoyo e influencia ascendente...); 4) trabajo en equipo y características sociales del ambiente (por ejemplo: cooperación, orgullo y calidez); y 5) atributos de la organización y el subsistema o departamento (entre otros, innovación, apertura a la información y sistema de recompensas y reconocimientos) (James y Sells, 1981; Jones y James, 1979). Cabe destacar que la mayoría de estas dimensiones han sido confirmadas en otros estudios (Mejías, Reyes y Arzola, 2006).

La evaluación del estado del arte realizada por Glick (1985) describía una lista abreviada de las dimensiones del clima, incluyendo la distancia psicológica al líder, la confianza gerencial (en el superior), así como la consideración, la comunicación, la apertura mental, la orientación al riesgo (tal como la concibieron Lawler, Hall, y Oldham, 1974), la calidad en el servicio (también considerada en estudios posteriores como los de Schneider, Wheeler, y Cox, 1992), la equidad y la centralidad. Después de la revisión de Glick (1985), el desarrollo de nuevas escalas ha continuado.

Koys y DeCottis (1991) incluyeron como dimensiones del clima organizacional: autonomía, cohesión, justicia, presión, innovación, reconocimiento, confianza y soporte. Davidson, Manning, Timo y Ryder (2001) tomaron en cuenta: soporte y facilidades del líder, espíritu profesional y organizacional, conflicto y ambigüedad, 
regulaciones de la organización y presión, variedad, reto y autonomía en el trabajo; además de estándares en el trabajo, cooperación, amistad y calidez en el trabajo en equipo.

Por su parte, Parker et al. (2003) a través de uno de los estudios más relevantes dentro del campo que nos ocupa y el cual fue realizado por medio de metanálisis, identificaron dimensiones comunes en las investigaciones del clima a nivel prácticamente mundial como: rol en el trabajo, el trabajo en sí, el líder, el grupo de trabajo, la organización en general, la satisfacción en el trabajo, el sentirse "bien" en el ambiente laboral, la motivación, el desempeño y otras actitudes hacia el trabajo.

En tanto Patterson et al. (2004 y 2005), basándose en una extensa revisión de la literatura, generaron un instrumento que consideró las siguientes dimensiones: involucramiento, autonomía, soporte del supervisor, integración, preocupación por el bienestar del empleado, desarrollo de habilidades, esfuerzo, reflexividad (capacidad para pensar en las decisiones laborales), innovación y flexibilidad, enfoque externo a la organización, claridad de metas, presión para producir, calidad (en el trabajo y de la empresa), retroalimentación del desempeño, eficiencia, formalización, tradición (seguir normas establecidas por años), afecto hacia los empleados, satisfacción general hacia el trabajo (respecto al superior, la organización y el puesto en sí), y dedicación en el trabajo.

Hernández Sampieri (2006) efectuó una revisión de la literatura con más de 50 estudios realizados en Estados Unidos y Europa entre 1960 y 2003, y seis en México; este autor encontró que las dimensiones más comunes son: percepción de la dirección-gerencia (apoyo experimentado), cooperación-trabajo en equipo, percepción sobre el desempeño-resultados-calidad, recompensas, autonomía, estructura, innovación, comunicación y motivación (principalmente intrínseca).

En resumen, podemos afirmar que tampoco se observa un consenso sobre qué variables deben medirse en cuanto al clima organizacional. Sin embargo, una vez más este panorama puede "iluminarse" con una operacionalización del clima organizacional a través de un modelo que lo contextualice y ubique, así como mida sus dimensiones (Patterson et al., 2005). Ahora bien, cabe aclarar que las visiones sobre el clima en las empresas e instituciones lo han vinculado con otro elemento importante: la cultura organizacional (por ejemplo, Robbins y Judge, 2010; D'Amato y Burke, 2009; Robbins, 2006). 
Por ahora, se abordará a la cultura organizacional y más adelante se ampliará la diferenciación y el vínculo entre el clima y la cultura organizacionales.

\section{La cultura organizacional}

La cultura organizacional es un recurso estratégico que posee el valor de asegurar la continuidad y permanencia de las organizaciones (Muro, 2008; Kaarst-Brown et al., 2004). Las culturas corporativas moldean la manera en cómo las personas actúan y se relacionan e influye fuertemente las formas en que se hace el trabajo. Abarca las metas de la organización, normas de conducta y las ideologías dominantes. La cultura puede expresarse a través de los mitos de la organización, sus héroes, leyendas, historias, jerga, ritos y rituales (Adeyoyin, 2006).

\section{Definiciones de cultura organizacional}

Peters y Waterman (1982) definieron a la cultura organizacional como un conjunto dominante y coherente de valores compartidos transmitidos por significados simbólicos como cuentos, mitos, leyendas, slogans y anécdotas. Las definiciones de la cultura organizacional nos refieren a las siguientes entidades (Yammarino y Dansereau, 2011; Virtanen, 2000):

- Una configuración única de normas, valores, creencias, asunciones, formas de comportamiento y aquellas características sobre la manera en la cual los individuos y grupos se combinan para hacer el trabajo.

- Un conjunto de significados -en gran medida tácitos- compartidos por un grupo de personas.

- Un patrón de premisas básicas compartido que el grupo aprendió para resolver los problemas de adaptación externa e integración interna.

- La conciencia invisible, implícita e informal de la organización que guía el comportamiento de los individuos.

- Los valores, creencias y actitudes relativamente estables y sostenidas que existen dentro de la organización.

El clima y la cultura organizacional: sus diferencias y vínculo

No hay duda que la cultura y el clima son conceptos relativamente semejantes, puesto que ambos describen las experiencias de los empleados con respecto a sus organizaciones; sin embargo, se trata de construcciones diferentes (Bunch, 2007). 
Svyantek y Bott (2004) proponen definiciones que nos ayudan a distinguir entre el clima y la cultura. La cultura organizacional es concebida como un conjunto de valores compartidos y normas sostenidas por los empleados que guían sus interacciones con compañeros, la dirección y los clientes. El clima está más orientado conductualmente; por ejemplo, los climas para la creatividad, la innovación, la seguridad o el servicio, pueden descubrirse en el lugar de trabajo.

Virtanen (2000) hace una reflexión proveniente de Denison (1996) sobre la distinción entre clima y cultura, que de acuerdo con gran parte de la literatura es la más aceptada: la cultura se refiere a la estructura profunda de las organizaciones, arraigada en los valores, creencias y premisas que son relativamente estables y son mantenidas por los miembros; mientras que el clima se encuentra arraigado en el sistema de valores organizacionales, sujeto a control directo y muy limitado a aquellos aspectos del ambiente social que son percibidos conscientemente por tales miembros.

Una cuestión básica para entender la diferencia entre ambos constructos en su origen, la cual es señalada por Denison (1996) y ubicada en los antecedentes teóricos. Los estudios de clima tienen sus raíces en la teoría de campo de Kurt Lewin, tal y como se comentó al hablar de las primeras investigaciones en la materia. Los estudios de cultura se fundamentaron en el interaccionismo simbólico y el constructivismo social. Debemos recordar que la cultura organizacional fue una derivación de la cultura en general, muy estudiada por los(as) antropólogos(as) como Margaret Mead. Los antecedentes iniciales del clima conducen a la concepción de que una persona es distinta de su contexto o ambiente social. Consecuentemente, el clima "es creado" por los gerentes (la dirección), y los empleados simplemente trabajan dentro de un clima pero "no lo crean". Los antecedentes de la cultura no separan al individuo de su ambiente; por lo tanto, la cultura es tanto el medio como el resultado de la interacción social. Denison (1996) efectuó una comparación entre ambos constructos, los cuales se muestran en el cuadro 1 . 


\section{Cuadro 1}

\section{Comparación entre la cultura y el clima}

\begin{tabular}{c|c|c}
\hline Foco & Cultura & Clima \\
\hline Epistemología & Contextual & Nomotética/ comparativa \\
\hline Punto de vista & De los nativos & Del investigador \\
\hline Metodología fundamental & Cualitativa & Cuantitativa \\
\hline Aspectos concernientes & Valores y premisas & Consenso en percepciones \\
\hline Fundamentos teóricos & $\begin{array}{c}\text { Teoría crítica/ } \\
\text { construcción social }\end{array}$ & $\begin{array}{c}\text { Psicología organizacional } \\
\text { más bien cuantitativa }\end{array}$ \\
\hline Disciplina básica & Antropología/ Sociología & Psicología \\
\hline
\end{tabular}

Extraído de Denison (1996).

Ahora bien, ambos constructos se encuentran relacionados porque, finalmente, las dimensiones del clima son significados, aunque puedan distinguirse. La cultura influye las orientaciones de las personas hacia los demás, el trabajo y el ambiente; mientras que el clima se muestra o despliega conforme los individuos experimentan calidez, ambigüedad y cuestiones similares a través de acciones e interacciones (Denison, 1996).

Históricamente, los estudios del clima se han traslapado con los de cultura en el sentido de que ambos tipos de investigación examinan el fenómeno organizacional (Denison, 1996 y Virtanen, 2000). Varios autores han señalado que el clima está más presente que la cultura, y ésta se encuentra más latente o subyacente que el clima (Virtanen, 2000). Patterson et al. (2005) consideran que el clima puede entenderse como una manifestación de la cultura; sin embargo, se requiere fortalecer la asociación entre los dos constructos, lo que puede hacerse mediante un modelo.

\section{Modelos de la cultura organizacional}

Para determinar las dimensiones más importantes de la cultura organizacional es fundamental utilizar un armazón o modelo teórico subyacente, que pueda reducir y enfocar la búsqueda de dimensiones culturales claves. Ningún modelo es completamente incluyente, ni totalmente correcto. Pero el modelo en el cual se fundamenten las investigaciones en el ámbito organizacional debe contar con soporte empírico, tratar de describir la realidad con exactitud (desde la perspectiva cuantitativa) y con profundidad (desde la óptica cualitativa); además, debe ser válido y que pueda integrar y organizar la mayoría de las dimensiones propuestas. 
Existen diversos modelos de la cultura, entre ellos el modelo de orientación cultural (Nakata, 2009; Hofstede y Hofstede, 2005), el modelo tridimensional de la cultura (Payne, 2001), el modelo de Schein (Schein, 2010) y el modelo de los valores en competencia (MVC) (Cameron y Quinn, 2011). De éstos, el que cuenta con mayor evidencia empírica (especialmente en América Latina) y posee mayor sencillez y claridad conceptual es, tal vez, el último (Maldonado et al., 2010; Yu y Wu, 2009; Muro, 2008; Quin y Rouhrbaugh, 2007; Cameron y Quinn, 2011).

\section{El modelo de los valores en competencia}

El MVC fue desarrollado inicialmente de la investigación realizada para encontrar los principales indicadores de las organizaciones efectivas a través de las preguntas como las siguientes: ¿cuáles son los criterios para determinar si una organización es o no efectiva?, ¿cuáles son los factores que definen la efectividad organizacional?; cuando un individuo juzga a una organización como efectiva, ¿qué indicadores tiene en mente? (Cameron y Quinn, 2011).

El modelo considera dos dimensiones/continuos: 1) flexibilidad, discreción y dinamismo versus estabilidad, orden y control; y 2) orientación interna, integración y unidad versus orientación externa, diferenciación y rivalidad. Los cruces de estas dos dimensiones producen cuatro subdominios culturales, tal y como se aprecia en la figura 1.

\section{Figura 1}

Modelo de los Valores en Competencia (MVC)

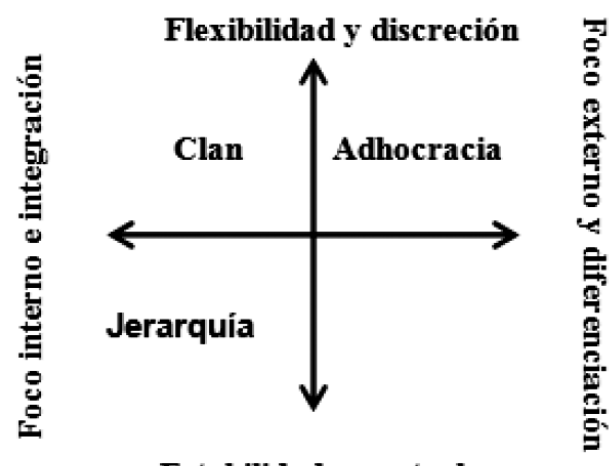

Estabilidad y control

Adaptada de Cameron y Quinn (2011) 
El modelo recibió su nombre porque los criterios dentro de los cuatro subdominios parecen llevar mensajes contradictorios. Los directivos quieren organizaciones adaptables y flexibles, pero también que a la vez sean estables y controladas. Los subdominios parecen ser cuatro perspectivas completamente diferentes, aunque están estrechamente relacionados y entretejidos; están compuestos de componentes con una estructura más grande: la efectividad organizacional y directiva. Los subdominios o cuadrantes en el MVC representan los valores subyacentes que se encuentran por encima de los empleados, programas, políticas y organizaciones. Los cuatro subdominios de cultura emergen dependiendo del tipo de organización; por lo tanto, la concepción de Cameron y Quinn (2011) implica clases de organizaciones ligadas a sus respectivas culturas. La mayoría de las empresas e instituciones posee elementos de todas las clases de culturas, pero desarrolla un estilo dominante; esto significa que no se trata de un modelo tipológico, sino topológico.

\section{- Clan}

Esta forma de organización recibe su nombre porque es muy similar al tipo de estructura familiar. Su concepción surgió después de estudiar a algunas firmas japonesas hacia el final de los años sesenta y principios de los setenta (Cameron y Quinn, 2011). Varios estudiosos observaron diferencias fundamentales entre los diseños organizacionales de jerarquía y mercado en Estados Unidos de América y compañías niponas, que se parecían a los clanes. La cultura fue definida en la denominada teoría Z de William Ouchi (Ouchi, 1982).

Cabe aclarar que metas y valores compartidos, cohesión, participación, individualismo colectivo y un sentido de nosotros permean a las empresas de tipo clan. Sus miembros se perciben más bien como una familia extensa que como entidades económicas (Cameron y Quinn, 2011). En lugar de reglas y procedimientos jerárquicos o las ganancias en los mercados, las organizaciones tipo clan se caracterizan por el trabajo en equipo, el involucramiento de los empleados en los programas y un compromiso corporativo con los trabajadores. El concepto de empowerment (empoderamiento) se aplicó a esta clase de organizaciones.

Las premisas básicas de la organización clan son: 1) el ambiente puede manejarse mejor a través del trabajo colaborativo y el desarrollo de los empleados; 2) los consumidores deben ser vistos como socios; 3) la organización está en el negocio de desarrollar un ambiente humano de trabajo; 4) la mayor tarea de la gerencia es otorgarles a los empleados el poder de decisión y facilitar su participación, dedicación, compromiso y lealtad. 
Asimismo, la cultura clan genera un clima amigable de trabajo donde los trabajadores comparten mucho de ellos mismos (Muro, 2008; Cameron y Quinn, 2011). Los líderes actúan como mentores y con elementos paternalistas. La organización se cimienta en la tradición y la lealtad; además de que a largo plazo enfatiza el beneficio del desarrollo individual, siendo importantes la cohesión y la moral. El éxito es definido en términos del clima organizacional y la preocupación por la gente. Elementos como el colectivismo (trabajo en equipo), la participación y el consenso resultan vitales.

\section{- Jerarquía}

Cameron y Quinn (2011) fundamentan esta clase de cultura en los atributos clásicos de la burocracia de Max Weber (reglas, especialización, meritocracia - supervisión mediante premios y sanciones - , jerarquía, propiedad separada, impersonalidad y responsabilidad), y en los trabajos clásicos de Henri Fayol y Frederick Winslow Taylor (Hernández Sampieri, 2010). Tales características fueron adoptadas por empresas e instituciones cuyo mayor reto fue generar eficiencia, confiabilidad, flujos planos, resultados predecibles. En los años sesenta representó el ideal de una compañía, debido a que la burocracia conducía a la estabilidad, la eficiencia y a una fuerte consistencia en la producción de bienes y servicios. Desde luego, el ambiente era relativamente estable y las tareas y funciones podían ser integradas y coordinadas; además, era posible mantener la uniformidad en los productos, los empleados y sus trabajos eran controlables. Las líneas claras en la toma de decisiones, la autoridad, las reglas estandarizadas y los procedimientos, así como el control y los mecanismos de responsabilidad fueron considerados como las llaves del éxito.

La cultura organizacional compatible con esta forma de organización se caracteriza por un lugar de trabajo estructurado y formalizado, y múltiples niveles jerárquicos; ahí los procedimientos gobiernan a los empleados; además, los líderes efectivos deben ser adecuados coordinadores y organizadores. Mantener a la organización en el largo plazo, sin turbulencia, es lo más importante. Asimismo, los aspectos centrales son la estabilidad, la predictibilidad, el control y la eficiencia, sin olvidar que las reglas formales y políticas mantienen a la organización unida (Cameron y Quinn, 2011; Yu y Wu, 2009).

La uniformidad de los productos o servicios resulta fundamental y los valores claves se enfocan en mantener la eficiencia, la fiabilidad, la rapidez y la producción constantes. Los nuevos empleados comienzan realizando un solo trabajo o tarea, 
los procesos están sujetos a tiempos y movimientos inflexibles; mientras que los ascensos requieren de un amplio conocimiento de las reglas y políticas.

- Adhocracia (ausencia de la jerarquía)

Este tipo de organización y la cultura asociada surgieron como consecuencia de la transición de la era industrial a la era de la información (Cameron y Quinn, 2011; Yu y Wu, 2009). Representa un diseño sensible a las condiciones aceleradamente cambiantes (turbulentas) que caracterizan al mundo organizacional del siglo XXI. Es resultado del rápido decrecimiento de la vida de los conceptos, productos y servicios. Una de sus premisas fundamentales es que las iniciativas innovadoras y pioneras conducen al éxito. Asimismo, "las organizaciones están principalmente en el negocio de desarrollar nuevos productos y servicios, y preparándose para el futuro" (Cameron y Quinn, 2011, p. 43).

La principal tarea directiva es lograr que se adopten la creatividad, el emprendimiento y la actividad de "permanecer en el límite". La adaptación y la innovación son vías para conseguir nuevos recursos y lograr la rentabilidad; consecuentemente, el énfasis se coloca en la creación de una visión del futuro, una "anarquía organizada" y una capacidad de imaginación considerable. Para Cameron y Quinn (2011) representa un diseño organizacional de reconstrucción permanente (cambia conforme nuevas tareas y productos son requeridos). Las adhocracias son temporales, se reconstituyen rápidamente cuando se presentan otras circunstancias. Una meta esencial de la organización adhocrática es crear adaptabilidad, flexibilidad y creatividad para combatir la incertidumbre, la ambigüedad y la carga excesiva de información, típicas del mundo globalizado.

Esta cultura se caracteriza por un lugar de trabajo creativo, dinámico y emprendedor; los empleados toman riesgos y el liderazgo efectivo es visionario, innovador y orientado a retos y amenazas. El lazo que mantiene a la organización unida es el compromiso con la experimentación. El énfasis empresarial se centra en ser vanguardista en cuanto a conocimiento, productos y servicios (Cameron y Quinn, 2011). El éxito se define en función de generar productos y servicios únicos y originales.

\section{- Mercado}

Constituye otra forma de organización surgida al final de los años sesenta, conforme las compañías enfrentaron nuevos retos (Cameron y Quinn, 2011). Su diseño 
es referido como una forma de mercado; sin embargo, el término mercado no es únicamente sinónimo de la función mercadológica, sino que va más allá, se refiere a un tipo de organización que funciona como mercado en sí misma. Está orientada hacia el ambiente externo en lugar de centrarse en los asuntos internos y se enfoca a las vinculaciones con las entidades del entorno como clientes y consumidores, proveedores, sindicatos, organismos reguladores y competidores.

La organización de mercado a diferencia de la jerárquica, donde el control interno se mantiene por las reglas, el trabajo especializado y las decisiones centralizadas; opera primariamente a través de mecanismos económicos y el intercambio monetario (Cameron y Quinn, 2011). Su mayor preocupación reside en conducir transacciones con los agentes externos como ventas, compras y alianzas tecnológicas (los contratos son esenciales). La rentabilidad, los resultados de intercambios, la fuerza en los nichos de mercado, la expansión de las metas y la lealtad del consumidor son los objetivos básicos de la compañía. Consecuentemente, los valores centrales son la competitividad y la productividad, los cuales se alcanzan mediante el posicionamiento externo y el control.

Las premisas fundamentales de la cultura de mercado son: a) el ambiente externo no es benigno sino hostil; b) los consumidores son sensibles y están interesados en el costo del producto o servicio (el valor agregado es importante); c) la compañía está inmersa en el negocio de incrementar su posición competitiva; y d) la tarea mayor de la gerencia es conducir a la organización hacia la productividad, los resultados y las ganancias. Para ello, se necesita de propósitos claros y una estrategia agresiva (Cameron y Quinn, 2011; Yu y Wu, 2009).

Los líderes deben ser asertivos, competitivos, pensantes y demandantes; el énfasis está en ganar y la preocupación en el largo plazo reside en las acciones competitivas y la ampliación de metas y objetivos (Cameron y Quinn, 2011). El éxito es definido en términos de participación y penetración del mercado; dejar atrás a la competencia y el liderazgo en el mercado resulta vital.

El modelo, además de especificar diseños organizacionales, culturas y valores, ordena los atributos de las organizaciones; debido a que fue formulado sobre la base de las premisas fundamentales acerca de cómo trabajan y son dirigidas las empresas e instituciones, describe otros aspectos organizacionales como los roles del liderazgo, los criterios de efectividad y las teorías administrativas asociadas más cercanamente con cada uno de los cuadrantes. 
La virtud del MVC no solamente reside en su abundante evidencia empírica, sino que incluye parte muy relevante de la historia del pensamiento administrativo y sus enfoques, y consecuentemente pueden considerarse diferentes variables del clima organizacional que reflejan los elementos de la cultura organizacional (los cuadrantes dentro del modelo), contextualizándolo y justificando la selección de ciertas dimensiones para su medición (la elección de variables es considerablemente racional); esto permite clarificar un nivel de teoría, de medida y de análisis para tal clima.

Con el fin de diagnosticar la cultura organizacional dentro del MVC suele utilizarse el cuestionario denominado Instrumento de evaluación de la cultura organizacional (OCAI ${ }^{1}$, por sus siglas en inglés (Cameron y Quinn, 2011; Muro, 2008), pero es una herramienta que mide en particular la mezcla de valores en las culturas actual (la que posee la organización) y preferida (la deseada por los miembros de la organización); en este sentido, nuestro trabajo pretende más que tal diagnóstico vincular al clima y la cultura organizacionales.

\section{Metodología}

La metodología y sus procedimientos se fundamentaron en la revisión de la literatura y el modelo de los valores en competencia. Este trabajo responde por sus características a una tradición metodológica del tipo cuantitativo.

\section{Hipótesis y variables}

Partiendo de los objetivos del estudio (relacionar al clima laboral con la cultura organizacional utilizando un marco de referencia apropiado y generar un instrumento que midiera las variables del clima laboral encuadradas en un modelo de cultura de organizacional) se establecieron las siguientes hipótesis que dirigieron esta investigación:

$H_{1}$ : De la medición de las variables del clima organizacional emergerán 17 dimensiones al efectuar la validación del constructo ${ }^{2}$.

\footnotetext{
${ }^{1}$ Organizational Culture Assessment Instrument.

${ }^{2}$ Número de variables incluidas en la medición y encuadradas en el MVC.
} 
$\mathrm{H}_{2}$ : Las unidades organizacionales de la muestra presentarán una mezcla de valores de todos los cuadrantes ${ }^{3}$, consecuentemente:

$H_{3}$ : Los cuadrantes pueden estar correlacionados entre sí; sin embargo:

$H_{4}$ : Las variables al interior de cada dominio o cuadrante tendrán correlaciones más altas con respecto a las correlaciones que pudieran tener con las dimensiones de otros dominios.

$H_{5}$ : Las organizaciones diferirán significativamente entre sí en cuanto a los cuadrantes o culturas.

Después de un proceso de validación por expertos (evidencia de validez de cara) ${ }^{4}$ y de acuerdo con la revisión de la literatura (evidencia de validez de contenido), 17 variables del clima organizacional fueron elegidas y enmarcadas dentro del MVC. A continuación se listan por cuadrante tales variables:

1) Cuadrante de cultura de clan (asociado con el enfoque administrativo de las relaciones humanas): bienestar de los empleados, autonomía, comunicación (en mayor medida para fines de involucramiento), énfasis en el entrenamiento, integración y soporte del superior inmediato.

2) Cuadrante de cultura jerárquica (vinculado con el enfoque administrativo del proceso interno): formalización y tradición.

3) Cuadrante de cultura u organización adhocrática (asociado con el enfoque administrativo de los sistemas): flexibilidad/innovación, enfoque externo y reflexividad.

\footnotetext{
${ }^{3}$ Tal y como lo predice el MVC.

${ }^{4}$ Dicha validación inicial incluyó a cuatro académicos con doctorado en Administración, dos gerentes de recursos humanos y un asesor en desarrollo organizacional. Las variables fueron seleccionadas por haber sido mencionadas en la literatura como parte de su respectivo cuadrante (por ejemplo, las variables de la cultura de clan han sido mencionadas en estudios de la escuela de relaciones humanas), particularmente en meta-análisis y revisiones del arte como Parker et al. (2003) — cuyo trabajo incluyó 121 muestras independientes de Estados Unidos, Reino Unido, India, Australia, Nueva Zelanda, Canadá e Israel—, Hernández Sampieri (2004) — quien revisó 52 estudios internacionales y cuatro trabajos nacionales - y Patterson et al. (2005), quienes consideraron a 42 empresas de manufactura. Desde luego, el estudio es exploratorio y la elección de variables representa un riesgo porque los análisis pueden confirmar o no su pertinencia. Los resultados subsecuentes nos indicarán si fue apropiada o no tal selección.
} 
4) Cuadrante que corresponde a la organización o cultura de mercado (que representa al enfoque administrativo de las metas racionales): claridad de metas organizacionales, esfuerzo, eficiencia, calidad, presión para producir y retroalimentación del desempeño.

\section{Instrumento de recolección de los datos}

Las variables fueron operacionalizadas a través de un cuestionario estandarizado, el cual se basó en dos instrumentos: 1) la medida del clima organizacional fundamentada en el modelo de los valores en competencia de Quinn y Rohrbaugh (Patterson et al., 2005); y 2) la escala Uni del clima organizacional validada para el medio laboral mexicano (Hernández Sampieri, 2006 y 2004). Las escalas son del tipo Likert con cuatro opciones de respuesta: dos positivas y dos negativas; por lo tanto, el rango potencial era de 1 a 4 , mínimo y máximo, respectivamente.

El instrumento inicial con 103 ítems fue sometido a un prueba piloto en una empresa de 120 empleados. Como resultado de la prueba (análisis de confiabilidad ${ }^{5}$ y análisis de factores por componentes principales $)^{6}$, se eliminaron ocho ítems, quedando un instrumento de 95 reactivos.

\section{Muestra}

La muestra fue heterogénea, en cuanto a rubro de organizaciones, tamaño y complejidad. Se incluyó a cuatro grandes empresas (de una se abarcó sólo a las oficinas corporativas) y cinco medianas, así como tres áreas funcionales de organizaciones (dos de servicios gubernamentales y una industrial) ${ }^{7}$, que son las siguientes:

\footnotetext{
${ }^{5}$ El valor alfa-Cronbach para toda la escala en la prueba piloto fue de 0.933 .

${ }^{6}$ El análisis mencionado se puede consultar en Hernández-Sampieri (2008).

${ }^{7}$ De acuerdo con la Ley para el Desarrollo de la Competitividad de la micro, pequeña y mediana empresa, publicada en el Diario Oficial de la Federación el 30 de diciembre de 2002 (última referencia publicada DOF 0606-2006); el Instituto Nacional de Estadística, Geografía e Informática (2004), los criterios de estratificación de empresas son los que se consignan en el siguiente cuadro:
}

\begin{tabular}{|c|c|c|c|}
\hline \multicolumn{5}{|c|}{ Clasificación por número de trabajadores } \\
\hline Sector / tamaño & Industria & Comercio & Servicios \\
\hline Micro empresa & $0-10$ & $0-10$ & $0-10$ \\
\hline Pequeña empresa & $11-50$ & $11-30$ & $11-50$ \\
\hline Mediana empresa & $51-250$ & $31-100$ & $51-100$ \\
\hline Gran empresa & A partir de 251 & A partir de 101 & A partir de 101 \\
\hline
\end{tabular}


1. Empresa comercial y de servicio dedicada principalmente a promover una tarjeta médica, con alrededor de 400 empleados, ubicada en el área metropolitana de la ciudad de México (muestra para el estudio, $n=326$ ).

2. Empresa industrial fabricante de bebidas no alcohólicas con 410 empleados, localizada en el área metropolitana de la ciudad de México (muestra para el estudio $n=333$ ).

3. Organización dedicada a promover un programa de lealtad para aficionados a un equipo de futbol con poco menos de 150 personas en nómina (área metropolitana de la ciudad de México (muestra para el estudio $n=120$ ).

4. Institución de educación básica e intermedia con 100 empleados administrativos y profesores, localizada en el estado de Querétaro (muestra para el estudio, $n=84$ ).

5. Empresa dedicada a brindar servicios de limpieza con presencia en todo México y 500 personas empleadas (muestra para el estudio, $n=67$, solamente se incluyeron las oficinas centrales de la Ciudad de México).

6. Firma de consultoría ubicada en la ciudad de México con alrededor de 75 personas (muestra para el estudio, $n=65$ ).

7. Empresa que fabrica autopartes con 150 individuos laborando en ésta y situada en el estado de Querétaro (muestra para el estudio, $n=113$ ).

8. Compañía de transporte ubicada en el estado de Guanajuato, con una plantilla de 100 personas (muestra para el estudio, $n=86$ ).

9. Empresa transportista ubicada en el estado de Guanajuato y con 70 empleados (muestra para el estudio, $n=50$ ).

10.Departamento de una empresa petrolera (el departamento está integrado por 500 personas y solamente nos dieron acceso a una $n=65$ ). El levantamiento se llevó a cabo en la Ciudad de México.

11.Departamento de un municipio del estado de Guanajuato (se entrevistó a todos los que laboran en éste, $n=48$ ). 
12.Agencia gubernamental desconcentrada del Estado de México, cuyo personal asciende a 190 empleados (sólo se pudo encuestar a 67 los cuales representan la $n$ ).

Tomando en cuenta que no todo el personal pudo participar por cuestiones de ausentismo o por negativa a responder la muestra de casos válidos fue de 1424 .

Finalmente, en este punto se reconoce que una limitante del estudio es la falta de información específica de los trabajadores que respondieron la encuesta (departamento, puesto, antigüedad, etc.), ya que en la práctica los mismos sienten cierto temor de ser identificados y afectados por las respuestas que marcaron en el instrumento de recolección.

\section{Resultados}

\section{Confiabilidad y validez del instrumento}

El proceso de validación fue el estándar que se acostumbra en estudios típicamente cuantitativos (Hernández Sampieri, Fernández y Baptista, 2010): determinación de la fiabilidad mediante el coeficiente alfa, análisis de factores por componentes principales (evidencia de la validez de constructo) y regresión múltiple (tomando como variables independientes a las variables del instrumento y como variable dependiente a la escala total, una medida predictiva).

El coeficiente alfa-Cronbach obtenido para todo el instrumento fue de 0.952 (con 95 ítems). Los coeficientes de confiabilidad para todas las variables se presentan en el cuadro 2. 


\section{Cuadro 2}

\section{Coeficientes alfa de fiabilidad para las dimensiones del clima organizacional}

\begin{tabular}{|c|c|c|c|}
\hline Dimensión & $\begin{array}{l}n \text { casos } \\
\text { válidos }\end{array}$ & $\begin{array}{l}\text { No. de } \\
\text { ítems }\end{array}$ & $\alpha$ \\
\hline Cuadrante de la cultura de clan (relaciones humanas) & 1138 & 40 & 0.95 \\
\hline 1) Bienestar & 1350 & 5 & 0.83 \\
\hline 2) Autonomía & 1376 & 6 & 0.77 \\
\hline 3) Comunicación & 1346 & 7 & 0.84 \\
\hline 4) Entrenamiento & 1388 & 4 & 0.61 \\
\hline 5) Integración & 1338 & 7 & 0.78 \\
\hline 6) Apoyo del superior & 1322 & 11 & 0.90 \\
\hline Cuadrante de la cultura jerárquica (proceso interno) & 1302 & 8 & 0.53 \\
\hline 7) Formalización & 1354 & 4 & 0.60 \\
\hline 8) Tradición & 1366 & 4 & 0.53 \\
\hline Cuadrante de la cultura adhocrática (sistemas) & 1294 & 17 & 0.87 \\
\hline 9) Innovación y flexibilidad & 1382 & 6 & 0.80 \\
\hline 10) Enfoque externo & 1352 & 6 & 0.70 \\
\hline 11) Reflexividad & 1368 & 5 & 0.67 \\
\hline Cuadrante de la cultura de mercado (metas racionales) & 1216 & 30 & 0.82 \\
\hline 12) Claridad de metas & 1370 & 5 & 0.79 \\
\hline 13) Esfuerzo & 1368 & 6 & 0.67 \\
\hline 14) Eficiencia & 1380 & 4 & 0.62 \\
\hline 15) Calidad & 1374 & 5 & 0.60 \\
\hline 16) Presión para producir & 1352 & 5 & 0.65 \\
\hline 17) Retroalimentación & 1388 & 5 & 0.68 \\
\hline
\end{tabular}

Como puede apreciarse en el cuadro 2, los coeficientes son muy aceptables, excepto por los referentes a la cultura jerárquica o proceso interno (pero todos son significativos al nivel del .05).

Una vez establecida la confiabilidad, se efectuó un análisis de factores por componentes principales para la validez de constructo de la escala total, los cuadrantes culturales y las variables (previamente se realizó la adecuación de la medida de KMO y la prueba de esfericidad de Barlett para ver que el análisis fuera apropiado, cuyos respectivos valores fueron de 0.918 y 48775.6 , ambos significativos). Los resultados resumidos de tal análisis se muestran en el cuadro 3. 


\section{Cuadro 3}

Análisis de factores por componentes principales Resultados del análisis de explicación de la varianza total ${ }^{8}$

\begin{tabular}{|c|c|c|c|c|c|c|}
\hline \multirow[b]{2}{*}{ Componente } & \multicolumn{3}{|c|}{ Variables eigen iniciales (eigenvalues) } & \multicolumn{3}{|c|}{$\begin{array}{c}\text { Extracciones de las cargas de las } \\
\text { sumas de cuadrados }\end{array}$} \\
\hline & Total & $\begin{array}{c}\% \text { de } \\
\text { varianza }\end{array}$ & $\%$ acumulado & Total & $\begin{array}{c}\% \text { de } \\
\text { varianza }\end{array}$ & $\%$ acumulado \\
\hline 1 & 22.596 & 23.785 & 23.785 & 22.596 & 23.785 & 23.785 \\
\hline 2 & 5.292 & 5.571 & 29.356 & 5.292 & 5.571 & 29.356 \\
\hline 3 & 3.686 & 3.880 & 33.236 & 3.686 & 3.880 & 33.236 \\
\hline 4 & 3.029 & 3.189 & 36.424 & 3.029 & 3.189 & 36.424 \\
\hline 5 & 2.306 & 2.427 & 38.851 & 2.306 & 2.427 & 38.851 \\
\hline 6 & 2.170 & 2.284 & 41.135 & 2.170 & 2.284 & 41.135 \\
\hline 7 & 1.924 & 2.025 & 43.160 & 1.924 & 2.025 & 43.160 \\
\hline 8 & 1.893 & 1.993 & 45.153 & 1.893 & 1.993 & 45.153 \\
\hline 9 & 1.615 & 1.700 & 46.853 & 1.615 & 1.700 & 46.853 \\
\hline 10 & 1.587 & 1.671 & 48.523 & 1.587 & 1.671 & 48.523 \\
\hline 11 & 1.505 & 1.584 & 50.108 & 1.505 & 1.584 & 50.108 \\
\hline 12 & 1.430 & 1.505 & 51.613 & 1.430 & 1.505 & 51.613 \\
\hline 13 & 1.389 & 1.462 & 53.076 & 1.389 & 1.462 & 53.076 \\
\hline 14 & 1.342 & 1.413 & 54.488 & 1.342 & 1.413 & 54.488 \\
\hline 15 & 1.260 & 1.326 & 55.814 & 1.260 & 1.326 & 55.814 \\
\hline 16 & 1.197 & 1.260 & 57.074 & 1.197 & 1.260 & 57.074 \\
\hline 17 & 1.161 & 1.222 & 58.297 & 1.161 & 1.222 & 58.297 \\
\hline 18 & 1.132 & 1.192 & 59.489 & 1.132 & 1.192 & 59.489 \\
\hline 19 & 1.123 & 1.182 & 60.671 & 1.123 & 1.182 & 60.671 \\
\hline 20 & 1.083 & 1.140 & 61.811 & 1.083 & 1.140 & 61.811 \\
\hline 21 & 1.032 & 1.086 & 62.897 & 1.032 & 1.086 & 62.897 \\
\hline
\end{tabular}

Elaboración propia a partir de los resultados del programa SPSS

\footnotetext{
${ }^{8}$ Por cuestiones de brevedad, presentación y enfoque de los resultados, se muestran únicamente las contribuciones de los 21 factores que emergieron con eigenvalores superiores a 1, no la estructura de 95 ítems. Los resultados completos se muestran en el apéndice 2 (matriz ítems por factores, cargas factoriales).
} 
La estructura obtenida fue de cuatro factores principales que contribuyen con el $36.4 \%$ de la varianza (sobre todo el primero: $22.6 \%$ ), dos menores y 15 secundarios, lo que presenta una consistencia con el modelo de los valores en competencia (se obtuvieron empíricamente los cuatro subdominios, cuadrantes o culturas) y las 17 dimensiones mencionadas previamente). Esto constituye una validación del MVC en función de elementos del clima organizacional y nos señala que la elección de variables fue, en términos generales, apropiada. ${ }^{9}$ La conclusión es que el instrumento en su conjunto demuestra validez de constructo.

\section{Caracterización de la cultura}

Asimismo, con la aplicación del instrumento sobre el clima organizacional vinculado a la cultura organizacional, se puede caracterizar a ésta de acuerdo con el MVC, utilizando los promedios en los cuadrantes (desde luego, contrastándolos mediante pruebas " $t$ ") y visualizándolos gráficamente.

\section{Discusión}

En primer término es necesario señalar que se cumplió con el objetivo de relacionar conceptualmente al clima laboral con la cultura organizacional dentro de un marco teórico lógico y congruente: El modelo de los valores en competencia (MVC). Tal asociación también se estableció, en términos generales, de manera empírica.

El usar el MVC para encuadrar al clima organizacional puede ser una manera de clarificar la relación entre éste y la cultura organizacional, resolviéndose consecuentemente la necesidad de un nivel de teoría, de medida y de análisis. Desde luego, se requieren más estudios en diferentes contextos y muestras diversas para ver si es el camino apropiado.

Del mismo modo, se desarrolló y validó un instrumento para medir el clima organizacional de acuerdo con la estructura de cuadrantes o culturas del MVC. La herramienta mostró ser sumamente confiable (estabilidad significativa) en su conjunto y en todos los cuadrantes o culturas, excepto en la de jerarquía o proceso interno (estabilidad media). Así, los resultados del análisis de factores (validez de constructo) revelaron que a través del instrumento se miden las cuatro culturas y sus respectivas variables, aunque de nuevo, el cuadrante de jerarquía no presenta el mismo nivel de validez.

${ }^{9}$ Las cargas de los ítems en los factores se presentan en el apéndice 2 (95 ítems por 21 factores). 
Lo anterior se debe probablemente a que sólo se utilizaron dos variables para medirlo. Resulta obvio que deben agregarse más variables; tal vez pudiera pensarse en construcciones como: centralización, complejidad, control, tensión, estrés, poder y gobernabilidad.

Con respecto a las hipótesis operacionales señalareros las siguientes conclusiones: por lo que se refiere a la primera cabe señalar que aunque se esperaban 17 factores (variables iniciales), se obtuvieron 21 , cuatro centrales y 17 que podríamos denominar secundarios; pero al efectuar un examen cuidadoso de la estructura de las matrices factoriales y los reactivos, es posible percatarse que mantienen un alto grado de correspondencia con el modelo de los valores en competencia (cuadrantes y dimensiones), lo que representa cierta validación empírica de éste y de las premisas claves de Patterson et al. (2005). Es decir, la hipótesis se corroboró, ya que emergieron las 17 variables, pero también surgieron las cuatro culturas como factores, lo cual fortifica la relación entre las variables del clima y el MVC (cultura organizacional); por otra parte, si se agregan variables a la cultura jerárquica, creemos que tal vínculo será mayor y el instrumento será todavía más válido y confiable.

En relación con la segunda y tercera hipótesis, derivadas de las consideraciones de Cameron y Quinn (2011) y la literatura que le antecede, podemos observar que han sido probadas en el contexto del estudio: las empresas de la muestra presentaron una mezcla de valores de todos los cuadrantes y no se concentraron en uno solo; esto se corrobora con el hecho de que los subdominios tuvieron correlaciones considerables entre sí, salvo en el caso del proceso interno. Lo anterior implica que la comprensión de la cultura de las empresas e instituciones debe basarse en el énfasis relativo que otorgan a cada uno de los valores en competencia (Cameron y Quinn, 2011; Patterson et al., 2005). Forzarlas para que sean caracterizadas en uno u otro cuadrante no podría representar la inherente dispersión de actividad y conocimiento de quienes laboran en ellas. Los empleados experimentan simultáneamente control y flexibilidad, así como enfoque interno y externo en sus empresas. Lo que resulta relevante para quienes estudian el clima y la cultura organizacional es el grado de énfasis, los tipos de prácticas gerenciales, así como la implementación real de las ideologías directivas.

En torno a la cuarta hipótesis, su demostración no quedó clara: las correlaciones al interior del cuadrante de la cultura de clan o relaciones humanas tienden a ser similares a las correlaciones entre las dimensiones de este cuadrante y los factores 
del subdominio de la cultura adhocrática; aunque las correlaciones al interior del cuadrante de la cultura de clan tienden a ser mayores a las correlaciones entre las dimensiones de éste y las variables del subdominio de la cultura de mercado. Este último patrón se repite al hablar de las dimensiones del cuadrante de la cultura adhocrática en comparación con los factores de la cultura de mercado.

Asimismo, podemos afirmar que el modelo de los valores en competencia es multidimensional y no lineal. Las construcciones de clima y cultura parecen ser más complejas de lo que se ha supuesto en la mayoría de la literatura organizacional: Paradójicamente son multidimensionales, pero resultan ser molares, en las cuales se funden distintas percepciones sobre aspectos centrales del ambiente de trabajo, la orientación directiva y los valores del empleado y la organización. Algo que en su momento apuntó Parker et al. (2003): detrás de las dimensiones del clima organizacional y otros conceptos se encuentra presente un proceso de juicio común, el cual se refleja en las distintas mediciones de la percepción del contexto laboral. Es un proceso subyacente que se expresa de diversas maneras.

El acuerdo entre participantes al interior de las organizaciones fue aceptable y mostró satisfacer las condiciones de agregación de percepciones entre los empleados. El hecho de que una medida efectivamente discrimine entre empresas e instituciones es muy importante, puesto que sugiere que las variaciones en el clima fueron reflejadas en los datos obtenidos por las escalas. Los estudios de factores organizacionales en el nivel de unidades completas deben poseer un alto grado de confianza, especialmente cuando los investigadores han identificado a priori organizaciones que difieren en cuanto a su naturaleza y características (Patterson et al., 2005; Parker et al., 2003).

En conclusión, sostenemos que el modelo de los valores en competencia y el instrumento perfilado son una forma adecuada y confiable para caracterizar la cultura de una organización y medir, simultáneamente, su clima. Tal modelo sigue demostrando solidez y se continua refinando, no solamente resulta útil para operacionalizar a la cultura organizacional, sino también para contextualizar al clima y su medición.

Las limitaciones del estudio son, desde luego, la muestra porque se trabajó únicamente con 12 organizaciones y 1424 casos, y el hecho de que la medición de la cultura jerárquica debe consolidar su confiabilidad y validez. Mientras que los resultados son de alcance causal, creemos que pueden contribuir a definir el rumbo 
para que otros estudios confirmen el modelo en distintas muestras de organizaciones latinoamericanas.

\section{Referencias}

Adeyoyin, S. O. (2006). Managing the library's corporate culture for organizational efficiency, productivity, and enhanced service. Library Philosophy and Practice 8 (2) [versión electrónica]. Disponible en: http://libr.unl.edu:2000/ LPP/adeyoyin.pdf.

Aralucen, P. (2003). Diseño y validación de un instrumento de medición del clima organizacional y la satisfacción laboral en una empresa avícola nacional. Tesis de maestría no publicada, Instituto de Estudios de Posgrado en Ciencias y Humanidades. México.

Brunet, L. (2002). El clima de trabajo en las organizaciones. 5a. reimpresión, México: Trillas.

Bunch, K. J. (2007). Training failure as a consequence of organizational culture. Human Resource Development Review 6 (2): 142-163.

Cameron, K. S. y R. E. Quinn (2011). Diagnosing and changing organizacional culture: Based on the Competing Values Framework, 3a. ed. San Francisco, CA: Jossey-Bass.

D'Amato, A. y M. J. Burke (2009). Psychological and organizational climate research: Contrasting perspectives and research traditions. A special issue of the European Journal of Work and Organizational Psychology. Londres: Psychology Press.

Davidson, M., M. Manning, N. Timo y P. Rider (2001). The dimensions of organizational climate in four-and five-star australian hotels. Journal of Hospitality \& Tourism Research 25 (4): 444-461.

Glick, W. (1985). Comment: Organizations do not cognize; response: Organizations are not central tendencies: Shadowboxing in the dark, round 2. Academy of Management Review 13 (1): 129-137. 
Hernández Sampieri, R. (2004). Validación de un instrumento para evaluar el clima organizacional en el medio laboral mexicano. Tesis de maestría no publicada, Instituto de Estudios Universitarios, Puebla, México.

(2006, septiembre). Validación de instrumentos para medir el clima organizacional. Documento presentado en el IV Congreso de Administración México, Centroamérica y el Caribe, Celaya, Guanajuato, México.

(2008). Exploración del modelo de los valores en competencia en el medio laboral mexicano. Disertación doctoral no publicada, Universidad de Celaya, Celaya, Guanajuato, México.

(2010, noviembre). El modelo de valores en competencia como reflejo de los enfoques administrativos. Documento presentado en el $1^{\circ}$ Congreso Internacional de Investigación. Trujillo, La Libertad, Perú.

, C. Fernández y P. Baptista (2010). Metodología de la investigación. 5a. ed. México: McGraw-Hill Interamericana Editores.

Hofstede, G. y G. J. Hofstede (2005). Cultures and organizations: Software of the mind. Ontario: The University of Western Ontario.

James, L. A. y L. R. James (1989). Integrating work environment perceptions: Explorations into the measurement of meaning. Journal of Applied Psychology 74 (5): 739-751.

James, L. R. y S. B. Sells (1981). Psychological climate: theoretical perspectives and empirical research. En D. Magnusson (ed.), Toward a psychology of situations: An interactional perspective (275-295). Hillsdale, NJ.: Erlbaum.

Johnson, B. H. (2009). Empowerment of nurse educators through organizational culture. Nursing Education Perspectives 30 (1): 8-13.

Jones, A. P. y L. R. James (1979). Psychological climate: Dimensions and relationships of individual and aggregated work environment perceptions. Organizational Behavior and Human Performance (23): 201-250. 
Kaarst-Brown, M. L., S. Nicholson, G. M. Von Dran y J. M. Stanton (2004) Organizational cultures of libraries as a strategic resource. Library Trends 53 (1): 33-53.

King, E. B., M. R. Hebl, J. M. George y S. F. Matusik (2010). Understanding tokenism: Antecedents and consequences of a psychological climate of gender inequity. Journal of Management 36 (2): 482-510.

Koys, D. J. y T. A. DeCottis (1991). Inductive measures of psychological climate. Human Relations 44 (3): 265-285.

Kuenzi, M. y M. Schminke (2009). Assembling fragments into a lens: A review, critique, and proposed research agenda for the organizational work climate literature. Journal of Management 35 (3): 634-717.

Lawler, E. E., D. Hall y G. Oldham (1974). Organizational climate: Relationship to organizational structure, process and performance. Organizational Behavior and Human Performance 11 (1): 139-155.

Litwin, G. y H. Stringer (1968). Organizational climate. Nueva York: Simon \& Schuster.

Maldonado, G., M. C. Martínez y D. García (2010). Aplicación del Modelo CVF en la pyme de Aguascalientes: Un estudio empírico. Investigación y Ciencia 50: 33-39.

Mamiseishvili, K. (2001). Characteristics, job satisfaction, and workplace perceptions of foreign-born faculty at public 2-year institutions. Community College Review 39 (1): 26-45.

Mathieu, J., M. T. Maynard, T. Rapp y L. Gilson (2008). Team effectiveness 19972007: A review of recent advancements and a glimpse into the future. Journal of Management 34 (3): 410-476.

McKnight, D. H. y J. Webster (2001). Collaborative insight or privacy invasion? Trust climate as a lense for understanding acceptance of awareness systems. En G. L Cooper, S. Cartwright y P. C. Earley (eds.), The International handbook of organizational culture and climate (533-555). Chichester, West Sussex: John Wiley \& Sons Ltd. 
Mejías, A., O. Reyes y M. Arzola (2006). Medición del clima organizacional en instituciones de educación superior. Universidad, Ciencia y Tecnología 10 (38): 55-61.

Muro, J. L. (2008). La cultura organizacional en la comunidad universitaria de la Universidad Pedagógica Nacional de Celaya. Disertación doctoral no publicada, Universidad de Celaya, Guanajuato, México.

Nakata, C. (2009). Beyond Hofstede: Culture frameworks for global marketing and management. Nueva York: Palgrave Macmillan.

Ouchi, W. (1982). Theory Z. Cambridge, MA: Avon Books.

Parker, C. P., B. B. Baltes, S. A. Young, J. W. Huff, R. A. Altmann, H. A. Lacost y J. E. Roberts (2003). Relationships between psychological climate perceptions and work outcomes: A meta-analytic review. Journal of Organizational Behavior 24 (4): 389-416.

Patterson, M., P. Warr y M. West (2004). Organizational climate and company productivity: The role of employee affect and employee level. Journal of Occupational and Organizational Psychology 77 (2): 193-216.

Patterson, M. G., M. A. West, V. J. Shackleton, J. F. Dawson, R. Lawthom, et al. (2005). Validating the organizational climate measure: Links to managerial practices, productivity and innovation. Journal of Organizational Behavior 26 (4): 379-408.

Payne, R. L. (2001). A three dimensional framework for analyzing and assessing culture/climate and its relevance to cultural change. En G. L Cooper, S. Cartwright, y P. C. Earley (eds.), The International handbook of organizational culture and climate. Chichester, West Sussex: John Wiley \& Sons Ltd: 107-121.

Peters, T. y R. Waterman (1982). En búsqueda de la excelencia. Nueva York: Harper \& Row.

Quinn, R. E. y J. Rohrbaugh (1983). A spatial model of effectiveness criteria: Towards a Competing Values Approach to organizational analysis. Management Science 29 (3): 363-377. 
Robbins, S. P. (2004). Comportamiento organizacional. 10a. ed. México: Pearson-Prentice Hall.

Robbins, S. P. y T. A. Judge (2010). Organizational behavior. 14a. ed. Upper Saddle River, NJ: Prentice-Hall.

Schein, E. H. (2010). Organizational culture and leadership. San Francisco, CA: Jossey-Bass.

Schneider, B. J., M. G. Ehrhart y W. H. Macey, (2011). Organizational climate research: Achievements and the road ahead. En En N. Ashkanasy, C. P. M. Wilderom y M. F. Peterson (eds.), The handbook of organizational culture and climate (29-49). Thousand Oaks, CA: Sage.

Schneider, B. J., J. K. Wheeler y J. F. Cox (1992). A passion for service: Using content analysis to explicate service climate themes. Journal of Applied Psychology 77 (5): 705-716.

Smith-Crowe, K., M. J. Burke y R. S. Landis (2003). Organizational climate as a moderator of safety knowledge-safety performance relationships. Journal of Organizational Behavior (24) 861-876.

Values, T. C. y H. Market (2005). The Competing Values Framework. International Journal of Manufacturing Technology and Management 2 (7): 10241040 .

Virtanen, T. (2000). Commitment and the study of organizacional climate and culture. En N. M. Ashkanasy, C. P. Wilderom, y M. F. Peterson (eds.). Handbook of organizational culture \& climate.Thousand Oaks, CA: Sage: 339-354.

Yammarino, F. J. y F. Dansereau (2011). Multilevel issues in organizational culture and climate research. En N. Ashkanasy, C. P. M. Wilderom y M. F. Peterson (eds.), The handbook of organizational culture and climate.Thousand Oaks, CA: Sage: 50-78.

Yu, T. y N. Wu (2009).A review of study on the Competing Values Framework. International Journal of Business and Management 4 (7): 37-42. 
